\title{
Labelling of Generalized Friendship, Windmill, and Torch Graphs with a Condition at Distance Two
}

\author{
Ikhsanul Halikin*, Hafif Komarullah
}

\author{
Graph, Combinatorics, and Algebra Research Group, Department of Mathematics, FMIPA, University of Jember \\ *Corresponding author. Email: ikhsan.fmipa@unej.ac.id
}

\begin{abstract}
A graph labelling with a condition at distance two was first introduced by Griggs and Robert. This labelling is also known as $L(2,1)$-labelling. Let $G=(V, E)$ be a non-multiple graph, undirected, and connected. An $L(2,1)$-labelling on a graph is defined as a mapping from the vertex set $V(G)$ to the set of nonnegative integer such that for $x, y \in V(G)$, $|f(x)-f(y)| \geq 2$ if $d(x, y)=1$ and $|f(x)-f(y)| \geq 1$ if $d(x, y)=2$, where $d(x, y)$ denoted the distance between vertex $x$ and $y$. The largest number of the vertex labels is called as span of $L(2.1)$-labelling. The span of a graph $G$ can be more than one, the minimum value of the span of a graph $G$ is notated by $\lambda_{(2,1)}(G)$. In this paper, we consider a graph labelling with distance two on generalized friendship, windmill, and torch graphs.
\end{abstract}

Keywords: L(2,1)-labelling, Labelling graph with distance two, Minimum of span, Generalized friendship, Windmill, and Torch graph.

\section{INTRODUCTION}

Graph labelling is an assignment from the elements of graph such as vertex, edge, or both to the set of nonnegative integer (commonly) with a certain requirement. Over the years, more than 200 graph labelling have been investigated. We can see it in [1] for further study. Every variety of graph labelling hold different condition. There are graph labelling with certain distance as a requirement, one of them is $L(2,1)$-labelling. An $L(2,1)$-labelling requires the difference between two vertex labels to reach certain conditions if the distance between two vertices is one or two [2].

Griggs and Roberts in the 1992 presented a concept of graph labelling with a condition at distance two. This concept is arisen from the modification of the frequency assignment problem presented by Hale in 1980. On a number of transmitters, each of them must be assigned a frequency to avoid frequency stacking. To overcome the frequency stack, then two transmitters that are "close" must receive different channels. While two transmitters that are "very close" must receive channels that are at least two channels apart. In the language of graph theory, the vertices of a graph represent transmitters, a "very close" transmitters represented by two vertices that are adjacent, and "close" transmitters represented by two vertices with distance two in the graph [3].
Let $G=(V, E)$ be a non-multiple, connected, and undirected graph. Suppose that $u, v \in V(G)$ and $d(u, v)$ denoted the distance between vertex $u$ and $v$. An $L(2,1)$ labelling of a graph $G$ is defined as a mapping $f: V(G) \rightarrow$ $\{0,1,2, \ldots, k\}$ such that $|f(u)-f(v)| \geq 2$ if $d(u, v)=$ 1 and $|f(u)-f(v)| \geq 1$ if $d(u, v)=2[2,4]$. A number $k$ such that an $L(2,1)$-labelling exist is called as span of $L$ (2.1)-labelling if there is no label greater than $k$. The span of a graph $G$ can be more than one, and the minimum value of the span of a graph $G$ is notated by $\lambda_{(2,1)}(G)[3]$.

There are many research about $L(2,1)$-labelling of a graph. Griggs and Yeh [2,5] in 1992 proved that $\lambda_{2,1}\left(S_{1, n}\right)=n+1, \lambda_{2,1}\left(C_{n}\right)=4$ and $\lambda_{2,1}\left(P_{n}\right)=4$. The minimum span of fan graph $\left(f_{n}\right)$ is $n+1$ and wheel graph $\left(W_{n}\right)$ is $n+1$ [6]. Yuri et al. [7] in 2018 proved that the minimum span of Sierpinski graph $\left(S_{(n, m)}\right)$ is 4 for $m=2$ and $m=3$.

In this paper, we present the minimum span of generalized friendship, windmill, and torch graphs. Before the further discussion, there are some properties of $L(2,1)$-labelling which will be used in this paper as follows.

Lemma 1.1. [8] If $H$ is a subgraf of graph $G$, then $\lambda_{2,1}(H) \leq \lambda_{2,1}(G)$. 
Lemma 1.2. [2] Let $S_{n}$ be a star graph with $n \geq 1$, then $\lambda_{2,1}\left(S_{n}\right)=n+1$.

\section{MAIN RESULT}

In this section we discuss about the minimal span of generalized friendship, windmill, and torch graphs.

\subsection{Generalized Friendship Graph}

Generalized friendship graph $\left(F_{n, m}\right)$ is defined as a collection of $m$ cycles $C_{n}$ with a common vertex [9]. Suppose that the vertices and edges of generalized friendship graph $\left(F_{n, m}\right)$ are notated as follows.

$V\left(F_{n, m}\right)=\left\{v_{0}, v_{j}^{i} ; i=1,2, \ldots, m ; j=1,2, \ldots, n-1\right\}$

$E\left(F_{n, m}\right)=\left\{v_{0} v_{1}^{m}, v_{0} v_{n-1}^{m}, v_{j}^{i} v_{k}^{i} ; j \neq k ; j, k=\right.$ $1,2, \ldots, n-1\}$

Its notation can be seen in Figure 1.

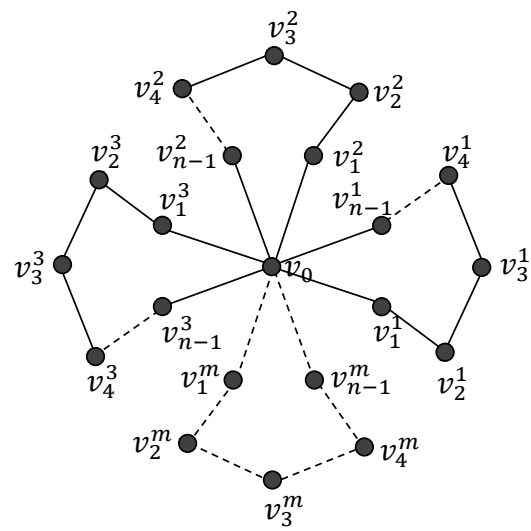

Figure 1 Generalized friendship graph $\left(F_{n, m}\right)$.

Theorem 2.1. Let $F_{n, m}$ be a generalized friendship graph with $n \geq 2$, and $m \geq 2$ then $\lambda_{2,1}\left(F_{n, m}\right)=$ $2 m+1$.

Proof. We will prove that $\lambda_{2,1}\left(F_{n, m}\right) \geq 2 m+1$. Since the star graph $\left(S_{2 m}\right)$ is a subgraph of generalized friendship graph $F_{n, m}$, then based on Lemmas 1.1 dan 1.2 , we have $\lambda_{2,1}\left(F_{n, m}\right) \geq \lambda_{2,1}\left(S_{2 m}\right)=2 m+1$. So, we have shown that $\lambda_{2,1}\left(F_{n, m}\right) \geq 2 m+1$. Next, we will prove that $\lambda_{2,1}\left(F_{n, m}\right) \leq 2 m+1$ by constructing $L(2,1)$ labelling on generalized friendship graph $F_{n, m}$. Let $f: V\left(F_{n, m}\right) \rightarrow\{0,1,2, \ldots, 2 m+1\}$ be a function as follows.

$$
\begin{gathered}
f\left(v_{0}\right)=0 \\
f\left(v_{1}^{i}\right)=2 i \\
f\left(v_{n-1}^{i}\right)=2 i+1
\end{gathered}
$$

For the image of $f\left(v_{j}^{i}\right)$ with $i=2, \ldots, m$ and $j=$ $2, \ldots, n-2$, we consider 2 cases as follows.

1. Cases 1: $m=2$

$$
\begin{aligned}
& \text { If } n \equiv 0 \bmod 3 \text { and } j=2, \ldots, n-2 \\
& f\left(v_{j}^{1}\right)=\left\{\begin{array}{l}
2 ; j=1 \bmod 3, j \neq n-2 \\
4 ; j=2 \bmod 3 \\
0 ; j=0 \bmod 3 \\
5 ; j=n-2
\end{array}\right. \\
& \text { If } n \equiv 1 \bmod 3 \operatorname{and} j=2, \ldots, n-2 \\
& \begin{array}{l}
4 ; j=1 \bmod 3, j \neq n-2 \\
2 ; j=2 \bmod 3
\end{array} \\
& \begin{array}{l}
0 ; j=0 \bmod 3 \\
3 ; j=n-2
\end{array} \\
& f\left(v_{j}^{1}\right)=\left\{\begin{array}{l}
2 ; j=1 \bmod 3, j \neq n-2 \\
4 ; j=2 \bmod 3 \\
0 ; j=0 \bmod 3 \\
5 ; j=n-2
\end{array}\right. \\
& f\left(v_{j}^{2}\right)=\left\{\begin{array}{l}
4 ; j=1 \bmod 3 \\
2 ; j=2 \bmod 3 \\
0 ; j=0 \bmod 3
\end{array}\right.
\end{aligned}
$$

$$
\begin{aligned}
& \text { If } n \equiv 2 \bmod 3 \text { and } j=2, \ldots, n-2 \\
& \qquad f\left(v_{j}^{1}\right)=\left\{\begin{array}{l}
2 ; j=1 \bmod 3, j \neq n-2 \\
4 ; j=2 \bmod 3 \\
0 ; j=0 \bmod 3 \\
1 ; j=n-2
\end{array}\right. \\
& f\left(v_{j}^{2}\right)=\left\{\begin{array}{l}
4 ; j=1 \bmod 3 \\
2 ; j=2 \bmod 3 \\
0 ; j=0 \bmod 3 \\
3 ; j=n-3 \\
1 ; j=n-2
\end{array}\right.
\end{aligned}
$$

Based on the definition of $L(2,1)$-labelling, two vertices with a distance one differ by at least two. It will be shown as follows. Since the labels of $\left\{v_{0}, v_{j}^{1}\right\}$ in the form of cycle sequence $(0,2,4,0,2,4, \ldots, 4,0,5,3)$ and the labels of $\left\{v_{0}, v_{j}^{2}\right\}$ in the form of cycle sequence $(0,4,2,0,4,2, \ldots, 0,3,5)$ for $n \equiv 0 \bmod 3$, it is easy to see that the difference labels of two vertices with distance one is at least two. In the same way, for the labels of $\left\{v_{0}, v_{j}^{1}\right\}$ and $\left\{v_{0}, v_{j}^{2}\right\}$ where $n \equiv 1 \bmod 3$ and $n \equiv$ $2 \bmod 3$, we can see that the difference labels of two vertices with distance one is at least two. Furthermore, for those of vertices with a distance two, we can also easily see that the difference of the labels is at least one. So, we can conclude that for $m=2$, the function $f$ satisfied $L(2,1)$-labelling. 
2. Case 2: $m \geq 3$

$$
\begin{aligned}
& f\left(v_{j}^{1}\right)=\left\{\begin{array}{l}
7 ; j=2 \bmod 3 \\
1 ; j=0 \bmod 3 \\
5 ; j=1 \bmod 3
\end{array}\right. \\
& f\left(v_{j}^{2}\right)=\left\{\begin{array}{l}
7 ; j=2 \bmod 3 \\
1 ; j=0 \bmod 3 \\
3 ; j=1 \bmod 3
\end{array}\right. \\
& f\left(v_{j}^{i}\right)=\left\{\begin{array}{l}
1 ; j=2 \bmod 3, i=3, \ldots, m \\
5 ; j=0 \bmod 3, i=3, \ldots, m \\
3 ; j=1 \bmod 3, i=3, \ldots, m
\end{array}\right.
\end{aligned}
$$

In the same way with the Case 1 , we can prove that the function $f$ satisfied $L(2,1)$-labelling for $m \geq 3$.

Since the function $f$ satisfied the rule of $L(2,1)$-labelling then we can conclude that $\lambda_{2,1}\left(F_{n, m}\right) \leq 2 m+1$. Therefore, we get $\lambda_{2,1}\left(F_{n, m}\right)=2 m+1$. We give an example to illustrate Theorem 2.1 in Figure 2.

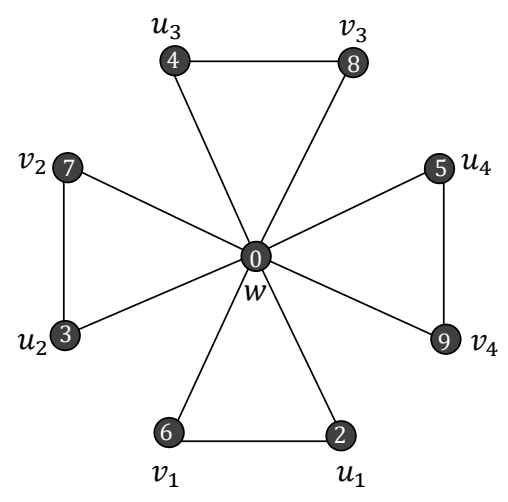

Figure $2 L(2,1)$-labelling of generalized friendship graph $F_{3,4}$.

\subsection{Windmill Graph}

Windmill graph $\left(W_{n}^{m}\right)$ is a graph obtained by combining $m$ copies complete graph $K_{n}$ with a common vertex. For the case $n=3$, windmill graph $\left(W_{n}^{m}\right)$ is a generalized friendship graph $F_{3, m}$. So, the windmill graph here is started with $n \geq 4$ and $m \geq 2$. Suppose that the vertices and edges of windmill graph are notated as follows.

$$
\begin{aligned}
& V\left(W_{n}^{m}\right)=\left\{v_{0}, v_{j}^{i} ; i=1,2, \ldots, m ; j=1,2, \ldots, n-1\right\} \\
& E\left(W_{n}^{m}\right)=\left\{v_{0} v_{j}^{i} ; i=1,2, \ldots, m ; j=1,2, \ldots, n-1\right\} \cup \\
& \left\{v_{j}^{i} v_{k}^{i} ; j \neq k ; j, k=1,2, \ldots, n-1\right\}
\end{aligned}
$$

The notation of vertices and edges of windmill graph as depicted in Figure 3.

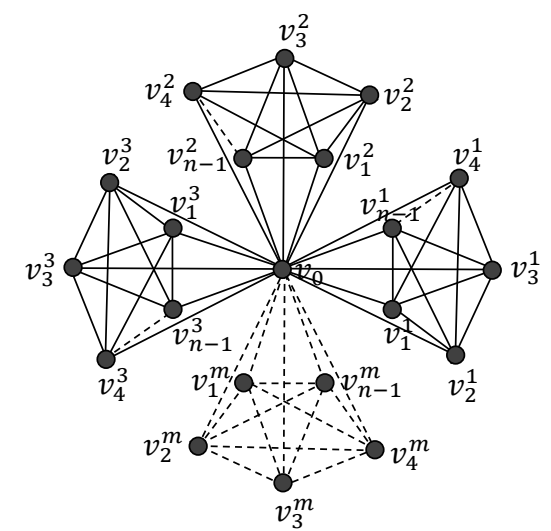

Figure 3 Windmill graph $W_{n}^{m}$.

Theorem 2.2 Let $W_{n}^{m}$ be a windmill graph with $m \geq 2$, then $\lambda_{2,1}\left(W_{n}^{m}\right)=\left\{\begin{array}{c}m n-m+n-1 ; m \text { is odd } \\ m n-m+1 ; m \text { is even }\end{array}\right.$

Proof. Let $W_{n}^{m}$ be a windmill graph with $m \geq 2$, we will consider two cases to prove this theorem.

a). Case 1: $m$ is odd

We will show that $\lambda_{2,1}\left(W_{n}^{m}\right) \geq m n-m+n-1$. Since the distance of vertex $v_{j}^{i}$ and $v_{k}^{i}$ for $j \neq k$ is one, then in order to satisfy the $L(2,1)$-labelling rule, the absolute value of their label difference is at least two. So, all vertices of $v_{j}^{i}$ and $v_{k}^{i}$ for $j \neq k$ must be labelled with even labels or odd label only. Since the distance of vertex $v_{j}^{i}$ and $v_{k}^{l}$ is two for $i \neq l$, then in order to satisfy a condition that $\left|f\left(v_{j}^{i}\right)-\left(v_{k}^{l}\right)\right| \geq 1$, every vertex of $v_{j}^{i}$ with $i=1,2, \ldots, m$ and $j=1,2, \ldots, n-1$ must be assigned different label. The minimal span of windmill graph $W_{n}^{m}$ can be reached if the label of vertex $v_{0}$ is 0 . Since vertex $v_{0}$ adjacent with every vertex $v_{j}^{i}$, then the label 1 cannot be attached to the vertex $v_{j}^{i}$. In view of $m$ is odd, all vertices $v_{j}^{i}$ need exactly $\left(\frac{m+1}{2}\right)(n-1)$ even labels and $\left(\frac{m-1}{2}\right)(n-1)$ odd labels. Considering that the minimum even label of vertices $v_{j}^{i}$ is 2 , then the largest label is $2+\left(\left(\frac{m+1}{2}\right)(n-1)-1\right) 2=m n-$ $m+n-1$. So, we have identified that $\lambda_{2,1}\left(W_{n}^{m}\right) \geq$ $m n-m+n-1$.

Next, we will prove that $\lambda_{2,1}\left(W_{n}^{m}\right) \leq m n-m+n-1$ by applying $L(2,1)$-labelling on windmill graph $W_{n}^{m}$. Define a function $f: V\left(W_{n}^{m}\right) \rightarrow\{0,1,2, \ldots, m n-$ $m+n-1\}$ as follows.

$$
f\left(v_{0}\right)=0
$$

For the image of all vertices $v_{j}^{i}$, there are two cases to be consider. 
1. If $i$ is odd

$$
\begin{aligned}
& f\left(v_{j}^{i}\right)=(m-1)(n-1)+2 j \\
& \text { for } j=1,2, \ldots, n-1
\end{aligned}
$$

2. If $i$ is even

$$
\begin{aligned}
& f\left(v_{j}^{i}\right)=(m-2)(n-1)+2 j+1 \\
& \text { for } j=1,2, \ldots, n-1
\end{aligned}
$$

Using similar method with Theorem 2.1, it can be easily seen that $f$ satisfied $L(2,1)$-labelling and we get $\lambda_{2,1}\left(W_{n}^{m}\right) \leq m n-m+n-1$. Therefore, we can conclude that $\lambda_{2,1}\left(W_{n}^{m}\right)=m n-m+n-1$.

\section{b). Case 2: $m$ is even}

We will prove that $\lambda_{2,1}\left(W_{n}^{m}\right) \geq m n-m+1$. Assume that windmill graph $W_{n}^{m}$ can be labelled by $0,1,2,3, \ldots, m n-m$. Since $v_{0}$ adjacent to all vertices $v_{j}^{i}$, then the minimum span of windmill graph $W_{n}^{m}$ can be obtained if the label of vertex $v_{0}$ is 0 . As a result, label 1 cannot be attach to any vertex of $v_{j}^{i}$ due to the rule of $L(2,1)$-labelling. Now, the remaining labels available are $m n-m-1$, while the number all vertices of $v_{j}^{i}$ is $m(n-1)=m n-m$. It means that we short of 1 label. Therefore, we need 1 label except labels $0,1,2,3, \ldots, m n-m$. So, the assumption that windmill graph $W_{n}^{m}$ can be labelled by $0,1,2,3, \ldots, m n-m$ is failed to be reached. Here, we have proved that $\lambda_{2,1}\left(W_{n}^{m}\right) \geq m n-m+1$.

Next, we will prove that $\lambda_{2,1}\left(W_{n}^{m}\right) \leq m n-m+1$ by formulating an $L(2,1)$-labelling on windmill graph $W_{n}^{m}$. Suppose that $f: V\left(W_{n}^{m}\right) \rightarrow\{0,1,2, \ldots, m n-m+1\}$ is a function with the rule as follows.

$$
f\left(v_{0}\right)=0
$$

For the map of all vertices $v_{j}^{i}$, we will divide it into two cases.

1. $\quad$ If $i$ is odd

$$
\begin{aligned}
& f\left(v_{j}^{i}\right)=(m-1)(n-1)+2 j \\
& \text { for } j=1,2, \ldots, n-1
\end{aligned}
$$

2. If $i$ is even

$$
\begin{aligned}
& f\left(v_{j}^{i}\right)=(m-2)(n-1)+2 j+1 \\
& \text { for } j=1,2, \ldots, n-1
\end{aligned}
$$

Again, in the same way with Theorem 2.1 it is easy to prove that $f$ is $L(2,1)$-labelling. So, we have $\lambda_{2,1}\left(W_{n}^{m}\right) \leq m n-m+1$. Therefore, we can conclude that $\lambda_{2,1}\left(W_{n}^{m}\right)=m n-m+1$. For example, an $L(2,1)$ labelling of windmill graph $W_{4}^{4}$ can be seen in Figure 4 .

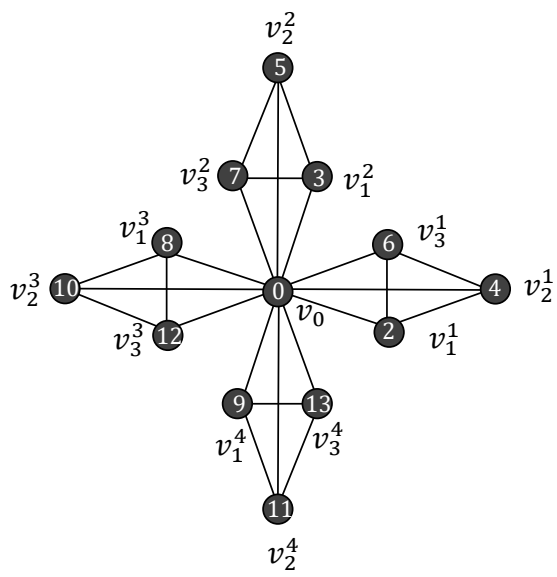

Figure $4 L(2,1)$-labelling of Windmill graph $W_{4}^{4}$.

\subsection{Torch Graph}

Torch graph $\left(O_{n}\right)$ is a graph obtained by combining a triangular book graph with a fan graph $f_{3}$ containing one leaf in the central vertex [10]. Suppose that the vertices and edges in the torch graph are notated as follows (see Figure 5 for the illustration)

$$
\begin{aligned}
V\left(O_{n}\right)= & \left\{v_{i} ; i=1,2, \ldots, n+4\right\}, \\
E\left(O_{n}\right)=\left\{v_{i} v_{n+1}, v_{i} v_{n+3} ; i=2, \ldots, n-2\right\} \cup & \left\{v_{1} v_{i} ; n \leq n \leq n+4 ; 1,2, \ldots, n-1\right\} \cup \\
& \left\{v_{n+3} v_{n+1}, v_{n-1} v_{n}, v_{n} v_{n+4}, v_{n} v_{n+2}\right\} .
\end{aligned}
$$

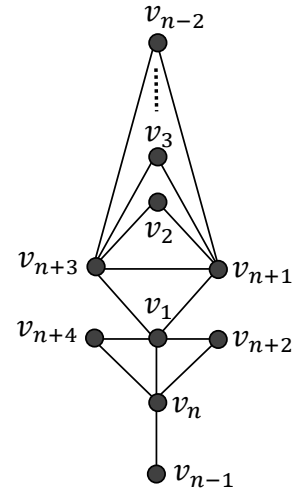

Figure 5 Torch graph $\left(O_{n}\right)$.

Theorem 2.3. Let $O_{n}$ be a torch graph with $n \geq 3$, then $\lambda_{2,1}\left(O_{n}\right)=\left\{\begin{array}{l}6 ; \text { for } 3 \leq n \leq 5 \\ n+1 ; \text { for } n \geq 6\end{array}\right.$.

Proof. Since the star graph and triangular book are the subgraph of a torch graph $O_{n}$ [11], it is easy to prove that $\lambda_{2,1}\left(O_{n}\right) \geq 6$ for $3 \leq n \leq 5$ and $\lambda_{2,1}\left(O_{n}\right) \geq \mathrm{n}+1$ for $n \geq 6$. 
Next, we will see that $\lambda_{2,1}\left(O_{n}\right) \leq \mathrm{n}+1$ by assigning $L(2,1)$-labelling on torch graph $O_{n}$. Let $f$ be a function from $V\left(O_{n}\right)$ to $\{0,1,2, \ldots, \mathrm{n}+1\}$ as follows.

$f\left(v_{i}\right)=\left\{\begin{array}{c}7-i ; i=1,2,3 \\ i+3 ; 4 \leq i \leq n-2 \\ i-n+1 ; i=n, n+1, n+2 \\ 4 i-4 n-12 ; i=n+3, n+4\end{array}\right.$

$f\left(v_{n-1}\right)=5$

It is easy to show that $\lambda_{2,1}\left(O_{n}\right) \leq n+1$. Then, we get $\lambda_{2,1}\left(O_{n}\right)=n+1$.

As an illustration, Figure 6 presents an $L(2,1)$-labelling of torch graph $\mathrm{O}_{6}$.

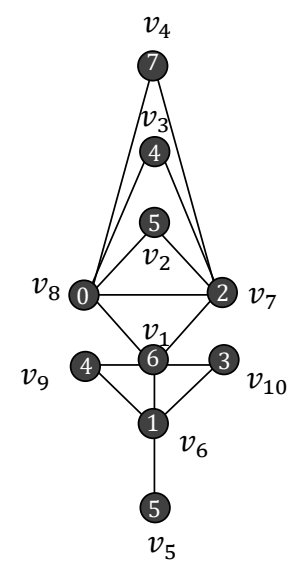

Figure $6 L(2,1)$-labelling of torch graph $O_{6}$.

\section{ACKNOWLEDGMENTS}

The first author was founded by "Hibah Fakultas Matematika dan Ilmu Pengetahuan Alam, Universitas Jember, Tahun Anggaran 2021, Nomor: 2461/UN25.1.9/KU/2021”.

\section{REFERENCES}

[1] J. A. Gallian, A dynamic survey of graph labeling, The Electronic Journal of Combinatorics, DS6, 2019. DOI: https://doi.org/10.37236/27
[2] J. Griggs, R. Yeh, Labelling graph with a condition at distance 2, SIAM J. Discrete Math, vol. 5, 1992, pp. 586-595. DOI: https://doi.org/10.1137/0405048

[3] Z. Shao, R.K. Yeh, D. Zhang, The $(2,1)$-Labeling on Graphs and Frequency Assigment Problem. Applied Mathematics Letters, vol. 21, 2008, pp.3741. DOI: https://doi.org/10.1016/j.aml.2006.08.029

[4] P. Bella, D. Král, B. Mohar, K. Quittnerová, Labeling planar graphs with a condition at distance two, European Journal of Combinatorics, vol. 28, Issue 8, 2007, Pages 2201-2239. DOI: https://doi.org/10.1016/j.ejc.2007.04.019

[5] R.K. Yeh, A survey on labeling graphs with a condition at distance two, Discrete Mathematics, vol. 306, Issue 12, 2006, pp. 1217-1231. DOI: https://doi.org/10.1016/j.disc.2005.11.029

[6] S. Fatimah, I.W. Sudarsana, S. Musdalifah, Pelabelan L(2,1) Pada Operasi Beberapa Kelas Graf (in Indonesian), Jurnal Ilmiah Matematika dan Terapan, 2016, 13(2), pp. 73-84.

[7] C. S. Yuri, Susiana, Pelabelan L $(2,1)$ pada Graf Sierpinski $S(n, k)$ (in Indonesian), Jurnal Sains Indonesia, 2018, 42(1), pp. 22-24. DOI: https://doi.org/10.24114/ jsi.v42i1.12244

[8] A. Lum, Upper Bound on L(2,1)-labelling Number of Graphs with Maximum Degree $\Delta, 2007$, https://www.whitman.edu/documents/academics/m athematics/lumaa.pdf

[9] H. Fernaua, J. F. Ryanb, K. A. Sugeng, A sum labelling for the generalised friendship graph, Discrete Mathematics, vol. 308, 2008, pp. 734-740. DOI:10.1016/j.disc.2007.07.059

[10] J. M. Manulang, K. A. Sugeng, Graceful labeling on torch graph, Indonesian Journal of Combinatorics, Vol 2, No 1, 2018, pp. 14-19. DOI: http://dx.doi.org/10.19184/ijc.2018.2.1.2

[11] Y. C. Sagala, Susiana, L(2,1)-labeling of Complete Multipartite Graphs, Proceedings of The 3rd Annual International Seminar on Trends in Science and Science Education, 2016, pp. 222-225. 